\title{
Ist die Heideggersche Philosophie des Seyns Mystik?
}

\author{
Łukasz KOŁOCZEK*
}

\begin{abstract}
Is Heidegger's philosophy of Being (Seyn) a mystique?: In this article I examine whether late Heidegger's philosophy (Gesamtausgabe volumes 65-74) can be called a mysticism. For serious reasons the answer should be negative. Heidegger's early statements relating to the mystique are very unflattering. I try to grasp what the Heidegger's exact attitude to the mystique is. Reflections on the mystique do not appear in late Heidegger's philosophy (with one exception). Heidegger treats the term "mysticism" as an objection to thinking, which consists in masking a lack of understanding of thinking. According to the immanent criteria, the philosophy of Being cannot be called the mystique. But it is possible to transpose the concept of the mystique, which means to carry the word from the first in another beginning. The second part of my work is an attempt at such a transposition. As a result I obtain the word "mysticism" conceived from the side of Being, that is as a source for mysticism conceived in the traditional way. So I can say that the philosophy of Being is a mystique in this particular sense of this word.
\end{abstract}

\section{KEYWORDS}

Martin Heidegger; mystique; philosophy; Being

* Doctor of Philosophy, independent scholar. E-mail: lukasz.koloczek@gmail.com. 
1. In diesem Artikel will ich die späte Philosophie von Martin Heidegger, die in den dreißiger und vierziger Jahren entstanden ist, aber erst am Ende des zwanzigsten Jahrhunderts in den Bänden 65 bis 71 der Gesamtausgabe veröffentlicht wurde, einer Prüfung unterziehen und untersuchen, ob man sie Mystik nennen kann. Dabei geht es nicht darum, Textstellen zu finden, in denen Heidegger den mutmaßlich mystischen Charakter seiner Philosophie erklärt, denn es gibt solche Stellen nicht. Im Gegenteil, ab und an äußert er sich sehr kritisch über Mystik. Es handelt sich hier auch nicht um eine Untersuchung der Quellen, aus denen Heidegger schöpft, und es soll auch nicht darauf hingewiesen werden, wie viel er den deutschen Mystikern schuldet, weil er nahezu aus der ganzen intellektuellen Überlieferung der abendländischen Zivilisation schöpft und man daraus, dass Heidegger etwas gelesen, zitiert, kommentiert hat, nicht schlussfolgern kann, dass er zu irgendeiner Tradition gehört. Ich werde die These vertreten, dass die Heideggersche Philosophie mystisch ist, trotz Heideggers Kritik an der Mystik. Meine Argumentation - das ist die dritte negative Bestimmung - betrifft nicht den Inhalt seiner Philosophie, das heißt, ich werde nicht beweisen, dass irgendeine der fundamentalen (oder nebensächlichen) Kategorien dieses Denkens dem Absoluten oder der Transzendenz entspricht und sich deshalb die Besinnung darauf als mystisch offenbart. Dem Gang der Darstellung vorgreifend und somit auch vereinfachend, kann ich vielmehr sagen, dass ich den mystischen Charakter dieser Philosophie in der Form suchen werde, die Heidegger seinem Denken gegeben hat.

Bevor ich mich dieser Aufgabe annehme, will ich den Bereich umreißen, in dem sich mein Versuch bewegen wird. Wenn wir von Mystik sprechen — ich wage die Behauptung: von irgendeiner Mystik in der abendländischen Welt bewegen wir uns in einem Bereich, der zwischen vier Faktoren aufgespannt ist. Ihre verschiedenen Modifikationen oder Gestalten stellen besondere Spannungen zwischen ihnen her. Ich denke hier neben der Mystik an die Theologie, die Geistigkeit und die Religion. Diese vier Begriffe sind keineswegs Synonyme, obwohl sie viel verbindet. Man kann keinen von ihnen mit einem der anderen gleichsetzen. Die Mystik lässt sich weder auf die Theologie, noch auf die Religion, noch auf die Geistigkeit zurückführen. Zugleich jedoch hängt wahrscheinlich jede Gestalt der Mystik mit irgendeiner Theologie, Religion und Geistigkeit zusammen. Wenn ich hier vorhabe, über den mystischen Charakter der Heideggerschen Philosophie nachzudenken, dann sollte ich darauf hinweisen, wie sie diese Faktoren entwirft. Zunächst untersuche ich daher kurz die Theologie, die Religion und die Geistigkeit, bevor ich im Hauptteil die Mystik einer Betrachtung unterziehen werde.

Heideggers Philosophie ist weder eine Theologie im allgemeinen noch in einem in der Tradition verwurzelten Sinne. Auf einen Slogan reduziert: Sie ist keine Wissenschaft über Gott. In den Texten von Heidegger zeigen sich zwar ziemlich oft Begriffe, die Gott in Erinnerung bringen können, insbesondere 
„der letzte Gott“ oder „die Götter“, Heidegger formuliert jedoch keinen Thesenkomplex, den man eine Theologie im traditionellen Sinn nennen könnte. Ähnlich wäre hier zu bestreiten, dass sie mit Geistigkeit im Sinne der Bevorzugung einer bestimmten Sphäre des Menschen - meistens des Geistes vor Anderem (also dem Körper) — verbunden wäre, weil diese Philosophie mit dem in eine bestimmte Sphäre im menschlichen Wesen ausgegliederten Denken programmatisch Schluss macht ${ }^{1}$. Also gibt es hier weder Postulate, sich vom Körper abzulösen, noch Ermunterungen, das Gemüt zu bilden, noch Aufforderungen, Aufklärung zu leisten. Schließlich ist diese Philosophie un-religiös, was hier bedeuten sollte: un-christlich, weil sie sich programmatisch außer und neben die Erfahrung des Glaubens stellt. Was den Inhalt der Heideggerschen Philosophie anbelangt (zwar nicht genau das Korpus, das ich untersuchen will, sondern die zu Lebzeiten publizierten Texte), dann wurden wahrscheinlich schon alle möglichen Meinungen über ihre Verschwägerung mit der Religion geäußert. Nur an äußerste Interpretationen erinnernd, will ich hier zum Beispiel Emanuel Lévinas erwähnen, der das Denken von Heidegger heidnisch nennt und es mit dem radikalen Monotheismus, wie er nur im Judaismus vorliegt, vergleicht (Lévinas, 1992). Hans Jonas interpretiert es als gnostisch (Jonas, 2001: 320-340). Man kann das Heideggersche Denken zum Atheismus zählen, aber es gibt auch Versuche, das Sein mit dem Gott der christlichen Theologie (in thomistischer Version) gleichzusetzen, was diesen Philosophen, wenn nicht zu einem Bekenner, dann wenigstens zu einem Verbündeten des Christentums macht ${ }^{2}$. Es genügt hier als Argument gegen solchen Schulterschluss an die Vielzahl von Stellen zu erinnern, wo Heidegger unter dem Christentum die Gestalt der abendländischen Metaphysik versteht.

Meine Meinung, die ich hier nur teilweise begründe, die aber meiner Lesart dieser Philosophie zugrunde liegt, stimmt mit keinem dieser Vorschläge überein, obwohl sie sich ähnlich anhört. Annäherungsweise sagt sie aus, dass die Heideggersche Philosophie ihrem Wesen nach christlich ist. Das bedeutet keineswegs, dass ich den oben signalisierten un-religiösen Charakter dieser Philosophie zurücknehme, noch dass ich dazu neige, das Sein oder Seyn mit dem Gott des Glaubens gleichzusetzen. Das lässt sich gewiß nicht machen, wenn man im Bereich dieser Philosophie bleiben will. Eher bin ich der Ansicht, dass

${ }^{1}$ Siehe Heidegger, 1975a: 324: „Die Verirrung des Biologismus ist dadurch noch nicht überwunden, daß man dem Leiblichen des Menschen die Seele und der Seele den Geist und dem Geist das Existentielle aufstockt und lauter als bisher die Hochschätzung des Geistes predigt, um dann noch alles in das Erleben des Leben zurückfallen zu lassen, mit der warnenden Versicherung, das Denken zerstöre durch seine starren Begriffe den Lebensstrom und das Denken des Seins verunstalte die Existenz".

${ }^{2}$ Ziemlich trotzig stellt Jacques Derrida die Vieldeutigkeit der Heideggerschen Philosophie und ihre möglichen oder unmöglichen Allianzen mit dem Christentum im letzten Kapitel seines Buches dar: Derrida, 1988. 
die Struktur dieser Philosophie, ihre innere Organisation, d.h. ihre Art des Fragens nach Fragwürdigem, ihrem Wesen nach christlich ist.

Ähnlich wie ich behaupte, dass die späte Philosophie Heideggers religiös in einem sehr besonderen Sinne ist, der mit dem umgangssprachlichen oder traditionellen Verständnis von Religion nichts zu tun hat, meine ich, dass sie eine Theologie in sehr besonderer Bedeutung ist. Heidegger erkennt in jeder Gestalt der Metaphysik, d.h. im Wesen der Metaphysik, zwei verschiedene Arten der Gründung des Seienden: erstens im Allgemeinsten — dementsprechend ist die Metaphysik immer Ontologie - und zweitens im Höchsten, dementsprechend ist sie immer Theologie. Heidegger zufolge, beweist die Geschichte der Metaphysik, dass, obwohl sie immer beide Arten der Gründung denkt, sie beide nicht in ihrer Einheit denken kann. Die Philosophie Heideggers ist ein Versuch, eine solche Einheit zu denken, was sie einerseits in das Wesen der Metaphysik selbst stellt (das sich in jeder Gestalt der Metaphysik verbirgt), aber sie andererseits zu einer Theologie, d.h. ursprünglichen Onto-theo-logie, macht. Das bedeutet nicht, dass nun der Gegenstand der Besinnung ein ursprünglicherer als der bislang gedachte Gott ist, sondern dass der Ursprung der beiden Arten der Gründung des Seienden gedacht wird ${ }^{3}$.

In einem sehr besonderen Sinne ist die Philosophie von Heidegger auch Geistigkeit. Der Begriff des Geistes, der in dieser Philosophie zugleich abwesend und allgegenwärtig ist, wie Derrida gezeigt hat, ist mit dem Feuer verbunden $^{4}$. In der Heraklit gewidmeten Vorlesung sagt Heidegger: „Das Feuer flammt und ist im Entflammen die Scheidung zwischen dem Lichten und dem Dunklen; das Entflammen fügt das Lichte und das Dunkle gegen- und ineinander" (Heidegger, 1994: 161). Wenn man also den Geist in einem der Metaphysik entgleitenden Sinn als das Feuer verstehen kann, dann besteht die Geistigkeit dieser Philosophie in solcher Verhaltenheit bei dieser Scheidung ${ }^{5}$, die ein Ursprung ist, dem erst der Unterschied zwischen dem Lichten und dem Dunkel entspringt, der aber beides in sich sammelt.

Sowohl Theologie als auch Geistigkeit werden im Rahmen der Strategie, die Metaphysik zu überwinden, gedacht und ausgesprochen, d.h. in Opposition zu jeder Bedeutung, die im Rahmen der Metaphysik zu denken möglich ist,

${ }^{3}$ Eine ausführliche Argumentation findet sich in meinem Buch (Kołoczek, 2013).

${ }^{4}$ Insbesondere Heidegger, 1985, aber auch in den Kommentaren zu Hölderlins Dichtung: Heidegger, 1981 und Heidegger, 1993 b.

${ }^{5}$ Am Rande will ich auf die Konsequenz dieses Denkens aufmerksam machen. In der Rektoratsrede erscheint die Definition des Geistes, nach der der Geist die „Entschlossenheit“ ist („[...] sondern Geist ist ursprünglich gestimmte, wissende Entschlossenheit zum Wesen des Seins“, Heidegger, 2000a: 112). In Beiträge zur Philosophie (Heidegger, 1989) wird „Entschlossenheit" auf „Entscheidung“ transponiert. Im Kommentar zu Heraklit ist der Begriff des Feuers mit der Idee der Scheidung verbunden. Wenn also der Geist als das Feuer und die Flamme gedacht ist, dann ist die Beziehung zwischen Geist und Entschlossenheit aufbewahrt, obwohl in einen anderen Anfang versetzt. 
und als der Ursprung dieser Bedeutungen. (Ähnlich verhält sich die Sache mit der möglichen, durchzuführenden Rekonstruktion eines unmetaphysischen Christentums). Die Absicht der Betrachtung des eventuell mystischen Charakters dieser Philosophie muss die besonderen Bedeutungen von Theologie und Geistigkeit berücksichtigen. Daher will ich nun aufzeigen, auf welche Art die Mystik mit der Metaphysik verstrickt ist, und dann versuchen, sie in einen anderen Anfang zu versetzen.

2. Heidegger denkt über das religiöse Leben in den Jahren 1919-1921 nach, und in diesem Zusammenhang finden sich ein paar Erwähnungen der Mystik ${ }^{6}$. Der ihr am ausführlichsten gewidmete Text enthält Notizen zu einem nicht gehaltenen Vortrag mit dem Titel Die philosopbischen Grundlagen der Mittelalterlichen Mystik (Heidegger, 1995: 303-337). Es ist sehr schwer, diese Notizen zu interpretieren, weil sie nur einen Entwurf des Vortrags darstellen, der zwar sehr detailliert ist, aber voller Gedankensprünge und Sätze, die erst während des Vortrags zu entfalten wären. Trotzdem findet sich hier ein Eintrag, der darauf hinweist, wie Heidegger Mystik versteht. Dieser Eintrag erklärt auch, warum der Philosoph gerade die Mystik betrachtet. In diesen Notizen über Mystik ist es nicht so wichtig, welche Inhalte die Mystik in sich trägt, weil der Gegenstand der phänomenologischen Untersuchung die mystische Erlebniswelt ist. Die Mystik ist hier nicht nur als eine emotionale Beziehung zum sacrum gedacht, sondern auch als eine breite Sphäre, in der man zwischen vier Bereichen unterscheiden kann:

I. Erlebnis („Leben“), II. Theorie des Erlebten (mystische Theologie) und seine theoretische, metaphysische Verwertung (religiöse, mystische Weltanschauung), III. Theorie des Erlebens selbst, IV. was sich mit I. eng und z. T. notwendig verbindet: erlebnismäßige Führung des Erlebens selbst [...] (Heidegger, 1995: 304).

Aufgrund der einleitenden Bestimmung der Mystik kann man sagen, dass sie freilich irgendwie eine Theorie dessen, was sich in der mystischen Erfahrung opfert, d.h des Gottes oder sacri, ist; die Mystik ist jedoch auch — nicht nur nachträglich, sondern wesentlich - eine Art, sich in der Welt zu befinden. Und es scheint für Heidegger am Interessantesten zu sein, dass die Mystik zugleich ein Wissen von dieser Art, sich in der Welt zu befinden, ist. Ähnlich betrachtet Heidegger anderenorts das ursprüngliche religiöse Leben, wenn er aufgrund der Paulus-Briefe die Existenz der ersten Christen einer phänomenologischen Analyse unterzieht. Die Phänomenologie wird hier selbstverständlich nicht als Methode der Analyse oder der Anleitung des Nachdenkens verstanden, sondern als die Methode „des durch die faktische Lebenserfahrung

\footnotetext{
${ }^{6}$ Sie sind in den folgenden Bänden versammelt: Heidegger, 1999; Heidegger, 1993a; Heidegger, 1995.
} 
führenden Zugangs“ (Heidegger, 1995: 34), und das grundlegende Phänomen, dem die Betrachtung sowohl über das religiöse Leben von Thessaloniker und Galater als auch über die mittelalterliche Mystik gewidmet ist, ist das Historische ${ }^{7}$. Heidegger nach, sollte man es nicht in der Geschichte der Philosophie suchen: „Wir können das Phänomen des Historischen aber nicht aus der Geschichtsphilosophie gewinnen, denn wir verwerfen die ganze Einteilung der Philosophie in Disziplinen. Damit ist das Historische gewissermaßen heimatlos geworden, es hat seinen systematischen Ort verloren. Wir müssen daher das Historische aus dem faktischen Leben entnehmen" (Heidegger, 1995: 34). Dieser Zusammenhang des Historischen mit dem faktischen Leben und die Phänomenologie als der Zugang zum Historischen durch das faktische Leben nehmen ein paar Jahre später in Sein und Zeit die Form der Analytik des Daseins an. Jetzt will ich nur hervorheben, dass die Besinnung sowohl auf das faktische Leben, als auch auf die Mystik in dem so umrissenen Horizont angesiedelt ist.

Die in Bänden 56/57 und 58 der Gesamtausgabe gesammelten Vorlesungen kreisen um die Philosophie - ihr Selbstbewusstsein und ihre ursprüngliche Erfahrung. Die Philosophie als Phänomenologie muss in der Beziehung zur Wissenschaft ${ }^{8}$ und zur Religion, also auch zur Mystik, begriffen werden. Letztere - kraft der umgangssprachlichen Bedeutung — ist der Wissenschaft gegenübergestellt (als Äquivalent dessen, was irrational, unklar, subjektiv ist) ${ }^{9}$. Wenn aber Heidegger seine Philosophie, d. h. Phänomenologie, in der Beziehung zu beiden bestimmen will, weist er darauf hin, dass sie in der Mitte zwischen Wissenschaft und Mystik liegt ${ }^{10}$.

${ }^{7}$ So lautet zum Beispiel der Titel von Paragraph 7.: „Das Historische als Kernphänomen“ (Heidegger, 1995: 31).

${ }^{8}$ In Heidegger, 1999: 18 behauptet er, dass „die Philosophie im Verlauf ihrer Geschichte immer in einem bestimmten Zusammenhang mit der Idee der Wissenschaft stand; einmal, in den Anfängen, war sie mit Wissenschaft überhaupt identisch; dann wurde sie, als $\pi \rho \omega ́ \tau \eta$ $\varphi 1 \lambda$ обофí $\alpha$, die Grundwissenschaft. In dem wesentlich praktisch gerichteten, aus der inhaltlichen Fülle seiner ihm aus allen Ländern zusammenströmenden Lebensmöglichkeiten oft mehr zum Selbstwert der Wissenschaft gelangenden Kulturzeitalter des Hellenismus tritt alle Wissenschaft überhaupt, und als Erkenntnis auch die Philosophie, in den Dienst unmittelbaren Lebens und wird zur Kunst der rechten Regelgebung für dieses. Im Zusammenhang mit der sich steigernden Herrschaft der moralischen und vor allem religiösen Lebenswelt und der ausnehmend geistig-seelischen Macht des entstehenden Christentums erhält sich die sekundäre, mittelhafte Stellung der Wissenschaft, die dann im Lebenssystem des Mittelalters zu einer Typischen reinen Ausprägung kommt. Die Zeit der Hochscholastik zeigt eine gewaltige Intensität des wissenschaftlichen Bewußtseins, das zugleich aber durchherrscht wird von der Kraft und Fülle der genuin forschenden religiösen Lebenswelt. Die ursprüngliche Motive und Tendenzen beider Lebenswelten laufen auf und fließen zusammen in der Mystik“.

${ }^{9}$ Siehe zum Beispiel Heidegger, 1993a: 149, 239.

${ }^{10}$ Heidegger, 1978: 410: „Besinnt man sich auf das tiefere, weltanschauliche Wesen der Philosophie, dann muß auch die Auffassung der christlichen Philosophie des Mittelalters als 
Die Mystik - Heidegger spricht hier von der mittelalterlichen Mystik und erwähnt an verschieden Stellen Bernhard von Clairvaux, Bonaventura, Eckhard, Tauler, Luther ${ }^{11}$ — hat in der Geschichte der abendländischen Philosophie eine besondere Stellung auch in dem Sinne, dass sie sehr streng Wissenschaft und Religion miteinander verbindet. Die Art dieser Verbindung erfordert ein paar kommentierende Worte.

In den frühen Schriften, in denen sich seine eigene Terminologie noch nicht ausgebildet hatte, spricht Heidegger vom Leben als dem ursprünglichen Bereich der Phänomenologie und von der Mannigfaltigkeit des faktischen Lebens.

Die faktische Lebenserfahrung ist etwas ganz Eigentümliches [...] Lebenserfahrung ist mehr als bloße kenntnisnehmende Erfahrung, sie bedeutet die ganze aktive und passive Stellung des Menschen zur Welt (Heidegger, 1995: 11).

Das Leben, die Erfahrung und die Welt vereinigen sich in einer Ganzheit: „»Welt « ist etwas, worin man leben kann“ (Heidegger, 1995: 11). In diesem Vortrag, aber auch in Grundprobleme der Pbänomenologie, unterscheidet Heidegger drei Momente der Lebenswelt: die Umwelt als der Moment des Lebens, der in dem Bezug auf Gegenstände besteht; die Mitwelt als der Moment des Lebens, der das Mitleben mit anderen Menschen umfasst; die Selbstwelt als der Moment des Lebens, der den menschlichen Bezug auf sich selbst bestimmt ${ }^{12}$. Seit ihren Anfängen erhält die Wissenschaft ihre leitende

im Gegensatz zur gleichzeitigen Mystik stehender Scholastik als prinzipiell verfehlt herausgestellt werden. Scholastik und Mystik gehören für die mittelalterliche Weltanschauung wesentlich zusammen. Die beiden »Gegensatz«-paare: Rationalismus-Irrationalismus und ScholastikMystik decken sich nicht. Und wo ihre Gleichsetzung versucht wird, beruht sie auf einer extremen Rationalisierung der Philosophie. Philosophie als vom Leben abgelöstes, rationalistisches Gebilde ist machtlos, Mystik als irrationalistisches Erleben ist ziellos“.

Heidegger, 1993a: 19: „[...] Mystik und Phänomenologie, die sowenig und soviel miteinander zu tun haben wie Phänomenologie und Botanik, Musik und Phänomenologie, bürgerliches Recht und Phänomenologie“.

Ähnlich in Heidegger, 1993a: 137 (und 231): „Die Strenge der philosophischen Wissenschaft ist nicht die-selbe wie die der mathematischen Naturwissenschaft, sie ist nicht die Strenge einer zwingenden Argumentation. Aber Philosophie ist deswegen nicht weniger streng als Mathematik; mathematische Strenge ist eben nicht Strenge schlechthin. - Strenge des philosophischen Ausdrucks besagt Konzentriertheit auf die Echtheit der Lebensbezüge im konkreten Leben selbst. Es handelt sich nicht um Mystik oder Schwärmerei, um ein Sich-loslassen und »Schauen«".

Heidegger, 1993a: 26: „Der »Ursprung« ist nicht ein letzter einfacher Satz, ein Axiom, aus dem alles abzuleiten wäre, sondern ein ganz Anderes; nichts Mystisches, Mythisches [...]” (das selbe in S. 203).

${ }^{11}$ Siehe zum Beispiel Heidegger, 1993a: 62, 205.

12 „Die Welt kann man formal artikulieren als U mwelt (Milieu), als das, was uns begegnet, wozu nicht nur materielle Dinge, sondern auch ideale Gegenständlichkeiten, Wissenschaften, 
Bestimmung aus der Umwelt ${ }^{13}$, und die Geisteswissenschaften können sie aus der Mitwelt schöpfen. Das bedeutet freilich nicht, dass alles, was in der Umwelt oder Mitwelt erscheint, zu „Materie“ für die Wissenschaft wird. Dafür ist eine Verwandlung der Einstellung zu den angetroffenen Sachverhalten in eine solche notwendig, die sie zum Gegenstand der Erkenntnis macht. Inzwischen gibt Heidegger Acht darauf, dass das faktische Leben sein Schwergewicht in die Selbstwelt verlagern kann.

Die Selbstbesinnung in mehr oder minder rohen Formen, das Leben in inneren Erfahrungen ist so alt wie die Menschheit - es ist Selbstbesinnung in der besonderen Gestalt der religiösen Nachdenklichkeit über Schicksal und die wirkenden, hemmenden und fördernden, strafenden und beglückenden Mächte des Lebens (Heidegger, 1993a: 56-57).

Das heißt nicht, dass nur die inneren Erlebnisse eine Bedeutung hätten und die Umwelt und die Mitwelt zu existieren aufhörten, sondern dass der beherrschende Sinn des im faktischen Leben Angetroffenen sich eher aus der inneren Erfahrung heraus bildet. Diese Art, den Gegenständen in der Welt zu begegnen, kann jedoch nicht objektiviert und deshalb nicht zum Ursprung der Wissenschaft werden ${ }^{14}$. Es geht hier meiner Meinung nach um die entscheidende Aufspaltung zwischen Wissenschaft und Religion. Am besten ist das im Christentum zu sehen, worin das Verlagern des Schwergewichts des faktischen Lebens in die Selbstwelt am tiefsten vollbracht wird ${ }^{15}$. Einerseits wird das Christentum (zumindest das frühmittelalterliche) zu einem Antagonisten der antiken Wissenschaft, vor allem der von Aristoteles; das zeigt sich besonderes bei Augustinus in seinen Confessiones und Soliloquia. Anderseits drückt sich aber das Christentum (im späteren Mittelalter) in der Sprache und Struktur

Kunst etc. gehören. In dieser Umwelt steht auch die Mitwelt, d. h. andere Menschen in einer ganz bestimmten faktischen Charakterisierung: als Student, Dozent, als Verwandte, Vorgesetzte etc. - nicht als Exemplare der naturwissenschaftlichen Gattung bomo sapiens $\mathrm{u}$. ä. Endlich steht auch das Ich-Selbst, die Selbstwelt, in der faktischen Lebenserfahrung “ (Heidegger, 1995: 11).

${ }^{13}$ Heidegger, 1993a: 46: „Diese neue merkwürdige Struktur des lebendigen Lebens an sich, daß sich in ihm alles irgendwie ausdrückt, gilt auch für die besonderen Ausformungen seiner Tendenzen, z. B. für die Wissenschaft. Diese nimmt ihren Ausgang in der Umwelt. Das ist ihr nächster Boden, auf den sie immer wieder in bestimmter Weise zurückgreift“.

${ }^{14}$ Heidegger, 1993a: 57: „Sie [d.h. verschiedene Formen der Autobiografie - E.K.] sind aber keine wissenschaftlichen Ausdrucksformen der Selbstwelt. Eine solche Objektivität zu beanspruchen, liegt gar nicht in ihrem Sinn. Sie nehmen die Ausdrucksmittel oft und meist aus dem eigenen Leben und seinen Erfahrungen selbst“.

${ }^{15}$ Heidegger, 1993a: 61: „Das tiefste historische Paradigma für den merkwürdigen Prozeß der Verlegung des Schwerpunktes des faktischen Lebens und der Lebenswelt in die Selbstwelt und die Welt der inneren Erfahrungen gibt sich uns in der Entstehung des Christentums“. 
dieser Wissenschaft aus ${ }^{16}$. Die mittelalterliche Mystik folgt selbstverständlich Augustinus und wird diesbezüglich aufgrund der Selbstwelt bestimmt. Sie kommt jedoch zum Wort, wenn sich die christliche Religion der wissenschaftlichen Sprache bedient ${ }^{17}$. In diesem Sinne stammt die Mystik von zwei Oppositionstendenzen des faktischen Lebens ab: von einer „biographischen“, auf der Selbstwelt gegründeten und von einer wissenschaftlichen, auf der Umwelt gegründeten. Man kann also sagen, dass sie sich zwischen der Wissenschaft (im aristotelischen Sinne) und der Mystik (in der umgangssprachlichen Bedeutung) platziert.

Die schon zitierte Struktur der Mystik, die das innige Erlebnis mit der Reflexion auf dieses Erlebnis vereinigt, scheint eine der Konsequenzen dieser Genese der Mystik zu sein, einer Genese, die beide Tendenzen des faktischen Lebens verbindet. Das ist, meiner Meinung nach, unter anderem der Grund dafür, dass das religiöse Leben und die Philosophie von Augustinus und auch die mittelalterliche Mystik Heidegger so sehr interessieren - denn sie nehmen eine ähnliche Position gegenüber dem faktischen Leben wie die Heideggersche Phänomenologie ein. Diese Koinzidenz der Phänomenologie und der Mystik soll die Unterschiede zwischen ihnen freilich nicht verhüllen, die Verortung im selben Bereich bedeutet nicht Identität. Ähnlich wie sich die Wissenschaft und die alltägliche Besorgung in demselben Bereich bewegen, was aber keinesfalls bedeutet, dass sie gleichgesetzt werden können ${ }^{18}$, so gibt es auch keine Gleichsetzung der Mystik mit der Phänomenologie. Die Phänomenologie ist darauf eingestellt, das Sein zu fassen, das sich in jedem Herausstellen des Seienden verbirgt. Heidegger untersucht jedoch den formalen Charakter der Mystik, um die Grundstrukturen der Phänomenologie zu fassen.

${ }^{16}$ Heidegger, 1993a: 61: „Die große Revolution gegen die antike Wissenschaft, vor allem Aristoteles, der aber gerade für das kommende Jahrtausend wiederum obsiegen, ja zu dem Philosophen des offiziellen Christentums werden sollte — in der Weise, daß die inneren Erfahrungen und die neue Lebensstellung in die Ausdrucksformen der antiken Wissenschaft gespannt wurden“.

${ }^{17}$ Heidegger, 1999: 18: „Im Zusammenhang mit der sich steigernden Herrschaft der moralischen und vor allem religiösen Lebenswelt und der ausnehmend geistig-seelischen Macht des entstehenden Christentums erhält sich die sekundäre, mittelhafte Stellung der Wissenschaft, die dann im Lebenssystem des Mittelalters zu einer typischen reinen Ausprägung kommt. Die Zeit der Hochscholastik zeigt eine gewaltige Intensität des wissenschaftlichen Bewußtseins, das zugleich aber durchherrscht wird von der Kraft und Fülle der genuin forschenden religiösen Lebenswelt“. Siehe Heidegger, 1993a: 212: „Im Christentum gelangt die Selbstwelt zu einer ganz neuen Bedeutsamkeit. Das zeigt sich philosophisch bei Augustin (Confessiones u. a.) und weiterhin im Mittelalter, besonders in der mittelalterlichen Mystik“.

${ }^{18}$ Heidegger nennt als Beispiel einen Arbeiter, der bei der Bauarbeit römische Münzen ausgegraben hat. Die Art, wie er den Gegenständen begegnet, ist ganz anders als die Art, wie ein Archäologe sie betrachtet. Siehe Heidegger, 1993a: 46-47. 
3. In einem späteren Zeitraum spricht Heidegger sehr selten über Mystik, ab und zu erwähnt er nur, sie sei eine Art von Erklärung, die nichts erklärend weitere Versuche zu verstehen schließt. Solche Ausflüchte können sowohl Heraklit (siehe Heidegger, 1994: 32) als auch Nietzsche ${ }^{19}$ betreffen. Manchmal beziehen sie sich aber auch auf das Denken des Seins ${ }^{20}$. Deshalb ist der 132. Paragraf der Besinnung so interessant :

Alle Mystik ist die von der Metaphysik selbst noch für sich oder gegen sich gesetzte Grenze.

„Mystik“ nur „innerhalb“ der Metaphysik, d. h. im Umkreis ihres Wesensbereiches; daher oft die Neigung, dem Anfang des abendländischen Denkens eine „Periode“ der „Mystik“ voraufgehen zu lassen, in der alles schon erfahren, was nachher die

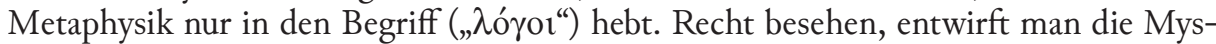
tik von der Metaphysik her. Dem entspricht die geschichtliche-metaphysische Rolle des Neuplatonismus, die Mystik des Mittelalters, die Mystik in der Zeit der Ausfaltung der neuzeitlichen Metaphysik und nocheinmal in der Romantik: Novalis, Baader, die Zweideutigkeit des Schellingschen Entwurfs und die negative und positive Philosophie. Sofern nun durch die seynsgeschichtliche Erfragung der Wahrheit des Seyns die Metaphysik als solche überwunden ist und die Möglichkeit der Verwendung metaphysischer Begriffe verwehrt, rettet man sich auf den Ausweg, das seynsgeschichtliche Denken als „mystisch“ zu bezeichnen; und man meint damit zugleich die abschätzige Feststellung von Unklarem und Dunklem, das Schwelgen in bloßen „Stimmungen“, jedenfalls ein Gebahren, das der „strengen“ Philosophie nie beachtungswert sein kann, vor dem aber die gelehrte Überwachung des historischen Philosophiebetriebes doch warnen muß.

Man merkt nicht, daß man so bereits selbst die Mystik zu einer Ab-und Unart der Metaphysik gemacht hat und weder die eine noch die andere im Wesen begreift.

„Mystik“ und Verrechnung des Seienden aus dem machenschaftlich gedachten Sein fordern sich wechselweise. Und in ihrer Eintracht vollbringen sie die Hintanhaltung des Übergangs aus der metaphysischen Geschichte des ersten Anfangs in den anderen; denn Übergang ist hier nicht die Stetigkeit eines Fortschreitens, sondern das Wissen der Jähe des Abbruchs zwischen Ende und Anfang (Heidegger, 1997: 403).

${ }^{19}$ Heidegger, 2000b: 123: „Entweder sagt man, dieser Gedanke Nietzsches sei eine Art »Mystik« und gehöre nicht vor das Denken [...] Was jedoch die erste Ausflucht angeht, nach der Nietzsches Gedanke von der ewigen Wiederkehr des Gleichen eine phantastische Mystik sei, so dürfte wohl das jetzige Zeitalter uns eines anderen belehren; gesetzt freilich, daß es dem Denken bestimmt ist, das Wesen der modernen Technik ans Licht zu bringen“ (dasselbe Heidegger, 2002: 112).

${ }^{20}$ Zum Beispiel Heidegger, 1975b: 72: „Denn das Geheimnis der Sprache, worin sich die ganze Besinnung versammeln muß, bleibt das denk- und fragwürdigste Phänomen, vor allem dann, wenn die Einsicht erwacht, daß die Sprache kein Werk des Menschen ist: Die Sprache spricht. Der Mensch spricht nur, indem er der Sprache entspricht. Diese Sätze sind nicht die Ausgeburt einer phantastischen »Mystik«. Die Sprache ist ein Urphänomen, dessen Eigenes sich nicht durch Tatsachen beweisen, das sich nur erblicken läßt in einer unvoreingenommenen Spracherfahrung“. 
In dieser Aussage erscheinen Feststellungen, die Heidegger schon früher geäußert hatte, also sowohl die, dass die Bezeichung des unverstandenen Denkens als „Mystik“ nur eine Flucht ist vor dem Versuch, dieses Denken zu verstehen (deshalb steht das Wort „Mystik“ in Anführungszeichen), als auch die, dass die Mystik eine gegensätzliche Tendenz zur Metaphysik ist, obwohl sie zugleich von ihr abstammt: „»Mystik« nur »innerhalb« der Metaphysik“. Jedoch wegen der zeitlichen Distanz zwischen dieser Aussage und früheren sowie der Evolution, die sich im Heideggerschen Denken zwischen dem Anfang der zwanziger und dem Ende der dreißiger Jahre ereignet hat, haben beide fast gleichlautenden Äußerungen einen ganz anderen Sinn. Anfänglich, während die eigene auf das Sein bezogene Terminologie von Heidegger noch nicht herausgearbeitet ist, ist der gemeinsame Raum für die gegenständlichen Tendenzen - Wissenschaft und Glaube, Wissenschaft und innige Erfahrung - das faktische Leben. Jetzt ist die menschliche Existenz nicht mehr der Bezugspunkt, sondern eben die Metaphysik — und zwar eine Metaphysik, die als Geschichte der Seinsvergessenheit und Seinsverlassenheit begriffen wird, weil sie als das Wesen der Metaphysik gedacht wird. Diese geschichtliche (wesentlich gedachte) Metaphysik beinhaltet auch die Mystik, und diese nimmt im Bereich ihres Ereignisses einen sehr wichtigen Platz ein. Die Mystik ist innerhalb der Metaphysik ihre Grenze. Das Wort „Grenze“ muss man richtig verstehen. Die Grenze ist hier nicht als eine Linie gedacht, die einen Bereich vom anderen, Metaphysik von Nicht-Metaphysik abtrennt. Die Grenze ist „das Innere“, d. h. sie gehört zum Wesen der Metaphysik. Als Grenze ist die Mystik zugleich eine Negation der Metaphysik, etwas für die Metaphysik Unwesentliches. Man soll das nach der „Logik“ der Zugehörigkeit des Unwesens zum Wesen denken ${ }^{21}$. Die Mystik sei ein unwesentliches Moment des Wesens der Metaphysik, wobei Unwesenheit nicht Unwichtigkeit bedeutet, sondern das notwendige Moment des sich Ereignens des Wesens selbst. Diese Figur kompliziert sich nachträglich dadurch, dass die Mystik nicht die einzige Gestalt des Unwesens der Metaphysik ist. Die erheblich öfter besprochene Gestalt des Unwesens ist das machenschaftlich gedachte Sein und das aufgrund dieser Auslegung gedachte Seiende im Ganzen. Diese Gestalt des Seins nennt Heidegger die Technik. Mystik und Technik gehören zueinander als die völlig verschiedenen Gestalten dessen, was von dem Wesen der Metaphysik abfällt, zwei gegensätzliche Gestalten des Unwesens der Metaphysik, zwischen denen sie selbst sich ereignet.

Im Lichte dieser Figur berechtigt das ab und zu bemerkte Heideggersche Interesse an den mystischen Schriften meiner Meinung nach nicht die Folgerung, dass Heidegger von der Mystik heimlich angezogen worden wäre und

${ }^{21}$ Diese „Logik“ könnte der Gegenstand für ganz andere Überlegungen sein. Zum Beispiel sieht man sie im solchen Fragment, der der Machenschaft gewidmet ist: „die Machenschaft das Unwesen des Seins begünstigt. Aber selbst dieses Unwesen ist, weil wesentlich dem Wesen, nie in eine Abwertung zu setzen“ (Heidegger, 1989: 126). 
deswegen auch heimlich nach einem Angleichen seiner Philosophie an die Mystik gestrebt hätte. Solche Versuche, diese Philosophie zu erklären, quittiert Heidegger mit der Formel: „[...] rettet man sich auf den Ausweg, das seynsgeschichtliche Denken als »mystisch « zu bezeichnen“. Inzwischen spielt die Mystik jeder Epoche eine wesentliche Rolle in der Geschichte der Metaphysik, sicherlich nicht im Vordergrund und gerade als deren Unwesen, aber dennoch eine wesentliche Rolle. Sollte ich diese Rolle rekonstruieren (was der Text erlaubt, auch wenn er sich nicht explizit dazu äußert), dann würde ich sagen, dass die Mystik als die von der Seinsvergessenheit, die die Form der alltäglichen Erfahrung des Seins annimmt, äußerst unterschiedliche Gestalt des Unwesens der Metaphysik mit ihrem Gegenteil einen Horizont absteckt, in dessen Rahmen man in jeder Epoche der Geschichte der Metaphysik das Sein denken kann. Das Aufzeigen des gegenüberliegenden Pols für die Philosophie hat jedoch vom Standpunkt der Geschichte des Seyns her noch einen anderen Sinn als nur das jedesmalige Aufzeigen des geschichtlichen Horizontes des denkbaren Seins. Die Mystik bewirtschaftet wirksam den Sinn der Negation gegen die Philosophie (z. B. wenn man die Philosophie für rational hält, wird die Mystik irrational). Von der Seite der Geschichte des Seyns her heißt das, dass der Übergang von der Metaphysik zu der Geschichte des Seyns unmöglich wird.

4. Die in diesem Fragment von Besinnung umrissene Diagnose scheint sehr aussagekräftig zu sein: die Mystik als das Andere der Metaphysik bildet den „Raum“ für ihre Geschichte, sie ist zugleich ein Schutz vor der Überwindung der Metaphysik. Als Negation der Metaphysik gehört die Mystik zur Metaphysik selbst und sie ist eine der metaphysischen Strategien, die den Sprung in den anderen Anfang verbietet. Eine der Konsequenzen dieser Erkenntnis, welche Rolle die Mystik in der Geschichte der Metaphysik spielt, ist das bereits zitierte Verbot, dem Denken des Seyns diesen Namen zu geben. Folgendes Argument scheint hier zu überzeugen: das Denken, in dem sich die Überwindung der Metaphysik mit allen ihren verschieden gedachten Grenzen, also auch mit der Mystik, vollzieht, kann nicht die Bezeichung eines der Elemente der zu überwindenden Metaphysik tragen. Das Denken in dem anderen Anfang als Mystik zu bezeichnen, wäre genauso unsinnig, wie es zum Beispiel als Technik zu bezeichnen.

Wenn ich trotzdem untersuchen will, ob das Denken des Seyns Mystik genannt werden kann, dann muss ich freilich auf das oben erläuterte Verständnis dieses Terminus verzichten. Der folgende Versuch wird sowohl eine Untersuchung der Heideggerschen Philosophie als auch eine Formulierung einer neuen, aus dem anderen Anfang her gedachten Bedeutung des Begriffes der Mystik sein. Zum Ausgangspunkt möchte ich die Frage nach dem Denken des Denkers selbst wählen, der an das Seyn in dem Raum zwischen dem ersten 
und anderen Anfang denkt. Wie charakterisiert Heidegger die Haltung des Denkers gegenüber dem zu Denkenden? Auf der ersten Seite der Beiträge zur Philosopbie steht bekanntlich, dass sich in diesem Denken ein „Wesenswandel des Menschen aus dem »vernünftigen Tier " (animal rationale) in das Da-sein“ vollbringen soll, was heißt „dem Er-eignis übereignet zu werden“ (Heidegger, 1989: 3). Auf diesen Wandel kann man selbstverständlich von verschiedenen Seiten blicken, unter anderem so, dass der Mensch, der sich dem Seyn zur Verfügung stellt, Wissen gewinnt: „[...] das rechte Begreifen dieser Verwandlung [...] ist zuinnerst verwoben mit dem Wissen der Wahrheit des Seyns" (Heidegger, 1989: 84). Das Wissen ist auch die Spur, auf die der schon zitierte Satz aus Besinnung bringt: „Übergang ist hier nicht die Stetigkeit eines Fortschreitens, sondern das Wissen der Jähe des Abbruchs zwischen Ende und Anfang". Das Wissen ist hier als der Übergang in den anderen Anfang gedacht, als der Übergang, den die Mystik und die Metaphysik zusammen untersagen. Also betrachte ich jetzt kurz, wie Heidegger das Wissen in Bezug auf den anderen Anfang versteht.

Der Begriff des Wissens hat im Entwurf des Seyns mehrere Dimensionen. Ohne alle aufzuzählen, kann ich einige erwähnen. Erstens ist das Wissen sehr streng mit der Philosophie verbunden: „Philosophie ist das nutzlose, gleichwohl herrschaftliche Wissen" (Heidegger, 1989: 36, siehe auch S. 43). Zweitens ist es gleich streng mit der Wahrheit verbunden, obgleich anders als im ersten Anfang: das Wissen ist die „Verwahrung der Wahrheit des Seyns“22. Diese Verwahrung bedeutet jedoch weder etwas in eine Tasche oder ein Geheimfach zu stecken noch etwas für später aufzubewahren, sondern eher eine Art des Schaffens: „[...] ein Wissen freilich, das nur als schaffendes in der Wahrheit steht. Schaffen — im weiteren Sinn hier gemeint — bedeutet jede Bergung der Wahrheit im Seienden“ (Heidegger, 1989: 24). Drittens ist das Wissen auch mit dem Da-sein verbunden: „Das Wesens-Wissen verlangt und ist selbst der Einsprung in das Da-sein“ (Heidegger, 1989: 287). Man kann abschließend noch einmal darauf hinweisen, dass das Wissen mit dem Übergang in den anderen Anfang $^{23}$ und mit der Überwindung des ersten Anfangs ${ }^{24}$ verbunden ist.

${ }^{22}$ Heidegger, 1989: 22. Siehe auch S. 71: „Wissen als Gründung der Wahrheit“.

${ }^{23}$ Heidegger, 1989: 172: „Was diese Frage [Was ist Metaphysik? — Ł.K.] erzielen will, ist nicht die Aufklärung und d. h. Festerhaltung der bisherigen und dazu notwendig verwirrten Vorstellung von der »Metaphysik«, sondern ist der Stoß in den Übergang und damit in das Wissen, daß jede Art von Metaphysik zu Ende ist und sein muß, wenn die Philosophie ihren anderen Anfang gewinnen soll“".

${ }^{24}$ Heidegger, 1989: 175: „Dieses Wissen ist, weil es den Nihilismus noch ursprünglicher in die Seinsverlassenheit hinabdenkt, die eigentliche Überwindung des Nihilismus, und die Geschichte des ersten Anfangs wird so völlig aus dem Anschein der Vergeblichkeit und bloßen Irre herausgenommen“. Siehe auch S. 219: „Um eine Bereitschaft zu schaffen für den Einsprung in das Da-sein, ist es daher eine unumgängliche Aufgabe, die Überwindung 
Für meine Argumentation ist jedoch wichtig, dass das Wissen mit der Frage nach dem Seyn eng zusammen hängt ${ }^{25}$. Zwar ist das Wissen im anderen Anfang immer noch das Wissen von... ${ }^{26}$, aber es ist keine Auskunft über etwas ${ }^{27}$. Im Heideggerschen Wörterbuch dominieren solche Bestimmungen des Wissens, die es mit dem Währen in der Haltung des Fragens vereinigen ${ }^{28}$. Zum Beispiel spricht der Paragraph 26 der Beiträge zur Pbilosopbie vom Wissen als vom Ursprung des Verzichtes:

Wenn das Wissen als Verwahrung der Wahrheit des Wahren (des Wesens der Wahrheit im Da-sein) den künftigen Menschen auszeichnet [... und ihn in die Wächterschaft für das Seyn erhebt, dann ist das höchste Wissen jenes, das stark genug wird, um der Ursprung eines Verzichtes zu sein (Heidegger, 1989: 62).

Der Verzicht bedeutet hier keine Schwäche ${ }^{29}$, sondern er wird als Bereitschaft für die Verweigerung bestimmt. Die höchste Gestalt des Verzichtes wird hier als der Ursprung der Bereitschaft gedacht, die zugleich die Bereitschaft zum Annehmen der Verweigerung, aber auch das Schaffen des „Raumes“ für das Denken an dem Seyn als der Verweigerung ist (es geht dagegen nicht um

des Platonismus einzuleiten durch das ursprünglichere Wissen um sein Wesen“; und S. 305: „Selbst die Ansetzung der i)de/a ist keine Ablesung! Dieses zu wissen heißt, sie überwinden“.

${ }^{25}$ Heidegger, 1989: 63: „Dieses Wissen entfaltet sich als das weitvorgreifende Fragen nach dem Seyn, dessen Fragwürdigkeit alles Schaffen in die Not zwingt und dem Seienden eine Welt errichtet und das Verläßliche der Erde rettet“. Zusätzlich ist das Wissen im Sprung in das Ereignis gedacht: „Wenn diese Verhaltenheit zum Wort kommt, ist das Gesagte immer das Ereignis. Dieses Sagen verstehen heißt aber, den Entwurf und Einsprung des Wissens in das Ereignis vollziehen“ (Heidegger, 1989: 80).

${ }^{26}$ Zum Beispiel: vom Ereignis (Heidegger, 1989: 13), vom Grund (S. 13: ,ihr Suchen liebt den Abgrund, in dem sie den ältesten Grund wissen"), vom Seyn (S. 117, 245), vom Unwesen (S. 39: "Das Wissen des Unwesens muß hier stets ein Vorbeigehen bleiben“), vom Wesen (S. 287: „Das Wesens-Wissen verlangt und ist selbst der Einsprung in das Da-sein“), von der Verweigerung (S. 63), von der Entscheidung (S. 102), vom anderen Anfang (S. 128, auch hier Wissen von der Zusammengehörigkeit der Machenschaft und des Erlebnisses), vom Nihilismus (S. 141: „Aber das Unumgänglichste und Schwerste in dieser Überwindung ist das Wissen vom Nihilismus"), um die Leitfrage und den Übergang (S. 232), von den Götter (S. 348), um den Zeit-Raurn als Abgrund (S. 388).

${ }^{27}$ Heidegger, 1989: 86: „Denn es gilt, keine neuen Vorstellungen vom Seienden zur Kenntnis zu bringen, sondern das Menschsein in die Wahrheit des Seyns zu gründen und diese Gründung im Erdenken des Seyns und des Da-seins vorzubereiten“. S. 287: „Daher kann es [Wissen — Ł.K.] durch bloße allgemeine Betrachtung des Gegebenen und seiner schon gefestigten Auslegung nie gewonnen werden“.

${ }^{28}$ Heidegger, 1989: 158: „Das Wissen aber ist die Inständigkeit in der Fragwürdigkeit des Seyns, das so seine einzige Würde wahrt, daß es sich selten genug verschenkt in der Verweigerung als das verborgene Ereignis des Vorbeigangs der Entscheidung über die Ankunft und Flucht der Götter im Seienden“.

${ }^{29}$ Einige Male sagt Heidegger, dass das Wissen „herrschend“ ist (Heidegger, 1989: 36, 43, $59,64)$. 
das sich dem Seyn Verweigern). „Das Wissen von der Verweigerung (Da-sein als Verzicht) entfaltet sich als die lange Vorbereitung der Entscheidung über die Wahrheit [...]“ (Heidegger, 1989: 63). Beide hier erscheinenden Bestimmungen: das Wissen als die Bereitschaft für die Verweigerung und als die Vorbereitung der Entscheidung ${ }^{30}$ ergeben sich nicht nur daraus, wie hier das Seyn gedacht wird (als Verweigerung und Entscheidung), sondern auch daraus, wie das Denken und der Denker gedacht werden. Im Kontext des Wissens zeigen sich noch andere Bestimmungen: Beständigkeit ${ }^{31}$, Inständigkeit ${ }^{32}$, Inständlichkeit $^{33}$, Sichhalten ${ }^{34}$, Bereitschaft, Vorbereitung, Wächterschaft ${ }^{35}$. Es ist klar, dass das Wissen in dem anderen Anfang sehr streng mit der Haltung des Denkers gegen das zu Denkende verbunden ist.

In einem der Paragrafen von Beiträge zur Philosophie vergleicht Heidegger das Wissen des anderen Anfangs mit dem Glauben. Im metaphysischen Denken wurde es Brauch den Verstand und den Glauben einander gegenüberzustellen. Heidegger hat gegen eine solche Auffassung dieser Frage schon früher protestiert, nämlich im Essay Pbänomenologie und Theologie (Heidegger, 1975b). Hier jedoch kompliziert sich die Sache, weil Heidegger an dieser Textstelle sagen kann, dass das Glauben im anderen Anfang dasselbe wie das Wissen ist. Die Struktur, nach der die Betrachtung hier angestellt wird, besteht in der Verdoppelung der Begriffe: also das Glauben im ersten und anderen Anfang und das Wissen im ersten und anderen Anfang, und weiter in dem Aufzeigen der sechs Verhältnisse zwischen ihnen.

a) Die Zusammenstellung des Glaubens mit dem Wissen im ersten Anfang ist übereinstimmend mit der Zusammenstellung der Metaphysik mit der Mystik in Besinnung:

Glauben meint aber, zumal in der offenen oder stillschweigenden Gegenstellung zum Wissen, das Für-wahr-halten dessen, was sich dem Wissen im Sinne der erklärenden

${ }^{30}$ Sowohl die Verweigerung als auch die Entscheidung sind Namen für die Wesung des Seyns.

${ }^{31}$ Heidegger, 1989: 287: „Da aber die Wahrheit gegründet werden muß im Da-sein, kann die Wesung des Seyns nur in der Beständigkeit errungen werden, die das Da aussteht in dem so bestimmten Wissen“.

${ }^{32}$ Heidegger, 1989: 158 (der Satz wird oben zitiert).

${ }^{33}$ Heidegger, 1989: 315: „Nur das, was wir, inständlich im Da-sein, gründen und schaffen und schaffend uns als Ansturm entgegentreten lassen, nur das kann ein Wahres, Offenbares sein und demzufolge erkannt und gewußt werden. Unser Wissen reicht nur so weit, als die Inständlichkeit im Da-sein ausgreift und d. h. die Kraft der Bergung der Wahrheit in das gestaltete Seiende“.

${ }^{34}$ Heidegger, 1989: 369: „Dieses Wissen ist [...] ursprünglich: das Sichhalten im Wesen der Wahrheit".

${ }^{35}$ Heidegger, 1989: 95: „Das Wissen von der stetigen Bedachtsamkeit des Seltenen gehört zur Wächterschaft für das Seyn, dessen Wesen als die Wahrheit selbst im Dunkel ihrer eigenen Glut erstrahlt". 
Einsichtnahme entzieht [...] Auch hier wird deutlich: dieses Glauben hängt in seiner Wesentlichkeit ab von der jeweils dagegen gestellten Weise des Wissens ${ }^{36}$.

b) Das Wissen - wie ich schon gezeigt habe — ist auch im Übergang gedacht, wodurch es eine ursprüngliche oder wesentliche Bedeutung bekommt:

Wenn das Wesen der Wahrheit ist: die Lichtung für das Sichverbergen des Seyns, dann ist Wissen: das Sichhalten in dieser Lichtung der Verbergung und somit der Grundbezug zum Sichverbergen des Seyns und zu diesem selbst (Heidegger, 1989: 369).

Der Übergang vom Wissen des ersten Anfangs zum Wissen des anderen Anfangs besteht in dem Sprung von den Vorstellungen über das Wahre zum Währen in der Wahrheit. c) Dieses Wissen kann in Bezug auf das Glauben des ersten Anfangs charakterisiert werden:

Dieses Wissen, das wesentliche Wissen, ist dann ursprünglicher als jedes Glauben, das immer nur auf ein Wahres geht und deshalb, wenn es überhaupt aus der völligen Blindheit heraus sein will, notwendig doch wissen muß, was ihm wahr und Wahres heißen! (Heidegger, 1989: 369)

Das wesentliche Wissen unterscheidet sich also wesentlich sowohl von dem Wissen des ersten Anfangs, als auch von dem Glauben des ersten Anfangs. d) Im Begriff und der Erfahrung des Glaubens befindet sich etwas, was verursacht, dass Heidegger sich für die Transposition dieses Begriffes vom ersten zum anderen Anfang entscheidet. Zuerst sagt er:

Das wesentliche Wissen ist ein Sichbalten im Wesen. Damit soll ausgedrückt sein: Es ist kein bloßes Vorstellen eines Begegnens, sondern das Aushalten innerhalb des Aufbruchs eines Entwerfens, das in der Eröffnung selbst den es tragenden Abgrund zu wissen bekommt (Heidegger, 1989: 369).

Und weiter: „Nimmt man daher »Wissen « im bisherigen Sinn des Vorstellens und Vorstellungsbesitzes, dann ist freilich das wesentliche Wissen kein »Wissen", sondern ein »Glauben" " (Heidegger, 1989: 369). Warum sollte das wesentliche Wissen ein „Glauben“ sein? „Allein, dieses Wort hat dann einen ganz anderen Sinn, nicht mehr den des Für-wahr-haltens, wobei Wahrheit schon, verworren genug, gewußt wird, sondern den des Sich-in-der-Wahrheit-Haltens" (Heidegger, 1989: 369). e) Das Wissen und das Glauben im anderen Anfang sind zwei Worte für dasselbe, nämlich für das Sich-in-derWahrheit-Halten. Deshalb ist das in den anderen Anfang transponierte Glauben auch das ursprüngliche Fragen, „in dem sich der Mensch in die Wahrheit

${ }^{36}$ Heidegger, 1989: 368. Die Ähnlichkeit besteht hier in dem Verweis gleichzeitig auf die Gegensätzlichkeit und auf die Abhängigkeit. 
hinaus und dem Wesen zur Entscheidung stellt“ (Heidegger, 1989: 369). Das Glauben „ist vielmehr das Ausharren in der äußersten Entscheidung“ (Heidegger, 1989: 370). Das ursprüngliche Glauben ist nicht mehr der Glaube an den Gott, sondern die Haltung des Denkers, das Sichhinausstellen in die Wesung des Seins ${ }^{37}$.

Die nach dem Seyn Fragenden - die im Rahmen dieses Entwurfs mit unterschiedlichen Namen bezeichnet werden: die Zu-künftigen, die Wenigen (die Seltenen), die Gründenden etc. — können jetzt auch „die ursprünglich und eigentlich Glaubenden" genannt werden:

Die Fragenden dieser Art sind die ursprünglich und eigentlich Glaubenden, d. h. diejenigen, die es mit der Wahrheit selbst, nicht nur mit dem Wahren von Grund aus ernst nehmen, die zur Entscheidung stellen, ob das Wesen der Wahrheit west und ob diese Wesung selbst uns, die Wissenden, Glaubenden, Handelnden, Schaffenden, kurz die Geschichtlichen trägt und führt (Heidegger, 1989: 369) ${ }^{38}$.

Die Transponierung des Glaubens in den anderen Anfang gibt ihm einen ursprünglichen Sinn - ursprünglich heißt hier: aus der Wesung des Seyns entspringend. Das ursprüngliche Glauben heißt jetzt das ursprüngliche Wissen, also Sich-in-der-Wahrheit-Halten.

5. Die Grundstimmung des anderen Anfangs ist die Verhaltenheit ${ }^{39}$. Diese Grundstimmung bildet ein Netz der Begriffe mit, die den Charakter des Denkens im anderen Anfang abstecken. Die Verhaltenheit bleibt in engem Zusammenhang mit den oben erwähnten Charakterzügen des Wissens: Bereitschaft, Vorbereitung, Wächterschaft. Sie ist auch mit der Inständigkeit verbunden, die eine der Grundzüge des Da-seins ist. Überdies gehen in den Bereich des Wandels des Daseins im Da-sein auch die Fügsamkeit ${ }^{40}$ und das zur VerfügungStellen ${ }^{41}$ ein. Es ist auch nötig die Analogie zwischen der glaubenden Existenz, die anhand der Analyse der Paulus-Briefe vorgestellt wurde, und dem Denken im anderen Anfang zu erwähnen.

${ }^{37}$ Heidegger, 1989: 370: „Denn dieses ursprüngliche Glauben ist auch kein eigensüchtiges Erraffen einer selbstgemachten Sicherheit, sofern es als Fragen sich gerade in die Wesung des Seins hinausstellt und die Notwendigkeit des Ab-gründigen erfährt“.

${ }^{38}$ Sehe auch Heidegger, 1989: 12: „Sie [die Fragende] sind die eigentlich Glaubenden, weil sie sich — eröffnend das Wesen der Wahrheit — auf den Grund zu halten“.

${ }^{39}$ Heidegger, 1989: 34: „Sie [die Verhaltenheit] ist die Grund-stimmung, weil sie die Ergründung des Grundes des Da-seins, des Ereignisses, und somit die Gründung des Da-seins stimmt“. Sehe auch S. 8 und S. 31.

${ }^{40}$ Heidegger, 1989: 309: „Der einrückende Entwurf aber entspringt nur aus der Fügsamkeit gegen die verborgenste Fugung unserer Geschichte in der Grundstimmung der Verhaltenheit“.

${ }^{41}$ Heidegger, 1989: 11: „Das Denken im anderen Anfang ist in einer einzigen Weise ursprünglich geschichtlich: die sich fügende Verfügung über die Wesung des Seyns“. 
Wenn ich jetzt die vierfache Struktur der Mystik (also das Erlebnis, die Theorie des Erlebten, die Theorie des Erlebens, die erlebnismäßige Führung des Erlebens) in Erinnerung rufe und auf sie vom anderen Anfang her blicke, dann eröffnet sich die Möglichkeit, die Mystik in den anderen Anfang zu transponieren. Die in der Historie vorkommende Mystik wurde im faktischen Leben gegründet, das auf das Ereignis des Christus (sein Leben, sein Tod und seine Auferstehung) bezogen ist, gleichwohl unterliegt sie dem Einfluss der mittelalterlichen Weltanschauung, die im wissenschaftlichen — im Sinne des Aristoteles - Bezug auf die Welt gegründet war. Als solche kann die Mystik in Besinnung als die Grenze der Metaphysik bezeichnet werden. Im anderen Anfang, der alle Weltanschauungen hinter sich lässt und als Grund aller Weltanschauungen die Metaphysik überwindet, sollte diese Zweispurigkeit der Mystik ihren Ursprung gewinnen. Die ursprünglich gedachte Mystik ist so kein Pseudobegriff mehr, der die Impotenz, das seynsgeschichtliche Denken zu verstehen, verhüllt, sondern die ursprüngliche Grundstimmung der $\mathrm{Zu}$-künftigen. Die Mystik im anderen Anfang soll die Einheit des Wissens und der Stimmung sein, das heißt die Einheit des Sich-in-der-WahrheitHaltens und des Währens in diesem Halten. Als solche ist die Mystik auch Bereitschaft für die Verweigerung, das heißt, für das Weghalten des Seyns. Dank ihrer Konstruktion, also: der Verhaltenheit in dem Sich-in-dem-Weghalten-des-Seyns-Halten, hält die Mystik — also die Philosophie — bei dem Seyn, dessen ausgezeichneter Charakter gerade die Verweigerung und Verbergung ist.

Auf diese Weise wurden alle vier Momente transponiert, die im ersten Anfang die Mystik bestimmen: das Erlebnis — in die Grundstimmung der Verhaltenheit, die Theorie des Erlebten - in den Entwurf des Seyns als des sich Verweigernden und des sich Verbergenden, die Theorie des Erlebens - in das Wissen und die erlebnismäßige Führung des Erlebens - in die Bereitschaft für die Verweigerung.

Die Umbildung der formalen Struktur der Mystik in die Form des Denkens des Seyns zieht noch andere Momente nach sich, die das mystische Denken im ersten Anfang gemeinsam erschaffen und im anderen Anfang aufbewahrt werden, obgleich sie sich nicht auf den in der mystischen Erfahrung begegnenden Gott beziehen, sondern auf das sich verweigernde und zum Denken gebende Seyn. Einige dieser Momente sind: Erstens, die Vereinigung des Mystikers mit Gott bekommt jetzt die Form des Da-seins. Das Da-sein ist sowohl die Gestalt, in die sich der Mensch gewandelt hat, als auch das Ereignis des Seyns. Zweitens, das Schweigen: das Wissen im anderen Anfang ist das Schweigen, ähnlich wie der Zuruf des Seyns. Drittens ist das Pathos des Heideggerschen Denkens sowohl dem Pathos der Paulus-Briefe ähnlich als auch dem der mystischen Aussagen. Dieses Pathos ergibt sich wahrscheinlich daraus, dass die ganze philosophierende Existenz dem Harren auf das Seyn und der 
Bereitschaft für dessen Verweigerung unterstellt wird. Zuletzt kann man die langsame Evolution im Entwurf des Seyns, nämlich von Beiträge zur Philosophie bis Das Ereignis, beobachten. Im Rahmen dieser Evolution verwandelt sich das Denken des Seyns, ohne mit dem Fragen nach dem Seyn aufzuhören, in das Hören auf das Seyn und in den Gehorsam. In Beiträge zur Philosophie wird diese Metapher immer noch in negativem Sinne benutzt: im ersten Anfang fehlt noch das Gehör für das Seyn ${ }^{42}$. Aber im 71. Band der Gesamtausgabe, wo der Entwurf des Seyns der immer weiter in den anderen Anfang verschobene Entwurf des anderen Anfangs zu sein scheint, wird das Seyn immer öfter durch das Wort „Gehorsam“ bestimmt ${ }^{43}$.

6. Das Denken des Seyns Mystik zu nennen, kann selbstverständlich für bloße Sophistik gehalten werden: so die Bedeutungen und den Sinn auszubeuten, um etwas zu sagen, wovor Heidegger warnt und empfiehlt, es nicht zu sagen. Es gibt jedoch im Werk Heideggers Beispiele für ein solches Umdefinieren von Wörtern. Ich denke hier zum Beispiel an das Wort „Glauben“, das zwar nicht zum Idiom des Seyns gehört, aber dennoch der Prozedur des Übergangs in den anderen Anfang unterzogen wird, um die an das Seyn Denkenden als Glaubende zu bezeichnen. Abgesehen davon kann man auf die Vorteile einer immanenten Ausarbeitung einer neuen Bedeutung des Begriffs „Mystik“ hinweisen. Der erste ist wahrscheinlich nicht der wichtigste. Ein solches Verständnis von Mystik lässt genauer eine gewisse Besonderheit begreifen, die man zwar auf anderen Wegen auch begreifen kann, die aber darauf hinweist, dass das Denken des Seyns theoretische und erlebnismäßige oder stimmungsmäßige Aspekte sehr eng in sich vereinigt. Dieses Denken ist nicht nur eine ,inhaltliche“ Strategie, die sich auf die Geschichte der Philosophie des Abendlandes bezieht, sondern auch ein existenzieller Entwurf, der ein Entwurf der Wandlung der Existenz in die Inständigkeit bei dem Seyn ist. Zweitens erscheint jedoch das Denken des Seyns auf diese Weise als der Ursprung, aus dem die traditionell begriffene Mystik entspringt (obwohl sie ihn zugleich verhüllt). Das Denken des Seyns kann man Mystik nennen, weil es der Ursprung aller Mystik ist. Wenn das so ist, dann werden alle Versuche, die Heideggersche Philosophie aus irgendeiner mystischen Tradition herzuleiten, - trotz ihrer historischen oder genetischen

${ }^{42}$ Heidegger, 1989: 73. Siehe auch S. 12, 243, 386.

${ }^{43}$ Heidegger, 2009: 48, 49, 102, 103, 177, 222, 255, 300, 332, 337. Siehe auch Heidegger, 1997: 337: „Die Seinsfrage fragt jetzt das Seyn, damit das Seyn antworte, das Wort verschenke, das die Wahrheit »des« Seyns sagt. Jetzt fragen wir nicht mehr am Sein vorbei, wobei das Seiende als solches das Fragliche bleibt, wir fragen auch nicht nur auf das Sein zu, wodurch das Sein nach der Art des metaphysischen Denkens zu einem »Gegenstand « wird, wir fragen das Seyn selbst. Das Fragen überantwortet uns dem Seyn als dem allein antwortenden. Solche Antwort verlangt ein anderes Hören aus einem gewandelten Horchen, das gehorsam ist aus einer vom Seyn selbst verschenkten Zugehörigkeit in die Wahrheit des Seyns“. 
Begründung - ihre Grundlosigkeit anerkennen müssen. Zwar besteht eine gewisse Analogie zwischen mystischem und seynsgeschichtlichem Denken, aber das Ereignis des Seyns macht die traditionelle Mystik möglich und diese ist eine der Weisen des Abfallens von ihrem Ursprung - eine andere als die Metaphysik, weil sie die „formalen“ und nicht die „inhaltlichen“ Züge des Ursprungs bewahrt.

\section{DIE BIBLIOGRAPHIE}

Derrida, J. (1988) Vom Geist. Heidegger und das Fragen. (Alexander Garcia Düttmann, Übers.). Frankfurt am Main: Suhrkamp.

Heidegger, M. (1975a). Brief über den Humanismus (S. 313-364). In: M. Heidegger. Wegmarken (=Gesamtausgabe, 9). Frankfurt am Main: Vittorio Klostermann.

Heidegger, M. (1975b). Phänomenologie und Theologie (S. 45-78). In: M. Heidegger. Wegmarken (=Gesamtausgabe, 9). Frankfurt am Main: Vittorio Klostermann.

Heidegger, M. (1978). Die Kategorien- und Bedeutungslehre des Duns Scotus (S. 189-412). In: M. Heidegger. Frühe Scbriften (=Gesamtausgabe, 1). Frankfurt am Main: Vittorio Klostermann.

Heidegger, M. (1981). Erläuterungen zu Hölderlins Dichtung (=Gesamtausgabe, 4). Frankfurt am Main: Vittorio Klostermann.

Heidegger, M. (1985). Die Sprache im Gedicht (S. 31-78). In: M. Heidegger. Unterwegs zur Sprache, (=Gesamtausgabe, 12). Frankfurt am Main: Vittorio Klostermann.

Heidegger, M. (1989). Beiträge zur Philosophie. Vom Ereignis (=Gesamtausgabe, 65). Frankfurt am Main: Vittorio Klostermann.

Heidegger, M. (1993a). Grundprobleme der Pbänomenologie (1919/20) (=Gesamtausgabe, 58). Frankfurt am Main: Vittorio Klostermann.

Heidegger, M. (1993b). Hölderlins Dichten des Wesens des Dichters als Halbgott (S. 153203). In: M. Heidegger. Hölderlins Hymne »Der Ister« (=Gesamtausgabe, 53). Frankfurt am Main: Vittorio Klostermann.

Heidegger, M. (1994). Der Anfang des abendländischen Denkens (S. 3-181). In: M. Heidegger. Heraklit (=Gesamtausgabe, 55). Frankfurt am Main: Vittorio Klostermann.

Heidegger, M. (1995). Pbänomenologie des religiösen Leben (=Gesamtausgabe, 60). Frankfurt am Main: Vittorio Klostermann.

Heidegger, M. (1997). Besinnung (=Gesamtausgabe, 66). Frankfurt am Main: Vittorio Klostermann.

Heidegger, M. (1999). Zur Bestimmung der Philosophie (=Gesamtausgabe, 56/57). Frankfurt am Main: Vittorio Klostermann.

Heidegger, M. (2000a). Die Selbstbehauptung der deutschen Universität (27. Mai 1933) (S. 107-117). In: M. Heidegger. Reden und andere Zeugnisse eines Lebensweges (=Gesamtausgabe, 16). Frankfurt am Main: Vittorio Klostermann.

Heidegger, M. (2000b). Wer ist Nietzsches Zarathustra? (S. 99-124). In: M. Heidegger. Vorträge und Aufsätze (=Gesamtausgabe, 7). Frankfurt am Main: Vittorio Klostermann.

Heidegger, M. (2002). Was heisst Denken? (=Gesamtausgabe, 8). Frankfurt am Main: Vittorio Klostermann.

Heidegger, M. (2009). Das Ereignis (=Gesamtausgabe, 71). Frankfurt am Main: Vittorio Klostermann. 
Jonas, H. (2001). Epilogue: Gnosticism, Existentialism, and Nihilism (S. 320-340). In: H. Jonas. The Gnostic religion. Boston: Beacon Press.

Kołoczek, Ł. (2013). Bóg Heideggera. Onto-teo-logiczny charakter „Przyczynków do filozofi””. Kraków: Dante.

Lévinas, E. (1992). Heidegger, Gagarin und wir (S. 173-176). In: E. Lévinas. Scbwierige Freibeit. Versuch über das Judentum. Frankfurt am Main: Jüdischer Verlag. 
